\title{
PUCES RÉCOLTÉES \\ DANS LA PROVINCE DE NEUQUÉN (ARgENTINE); DESCRIPTION DE 4 NOUVEAUX TAXA (INSECTA, SIPHONAPTERA)
}

\author{
J. C. BEAUCOURNU*, J. A. ALCOVER**/***
}

RÉSUMÉ. La récolte de 973 puces de décembre 1987 à mai 1988 dans la province de Neuquén (Argentine) sur divers petits mammifères terrestres a livré 20 taxa. Hectopsylla pascuali n. sp., Plocopsylla consobrina n. sp., Agastopsylla boxi gibbosa n. ssp., Chiliopsylla allophyla tonnii n. ssp. sont décrits. Plocopsylla diana Beaucournu, Gallardo et Launay, 1986, Ctenoparia inopina a Rothschild, 1909, Neotyphloceras crassispina chilensis Jordan, 1936, Tetrapsyllus satyrus Beaucournu et Torrés-Mura, 1986, Ectinorus martini Lewis, 1976, sont nouveaux pour l'Argentine.

Mots-clés : Puces. Siphonaptera. Espèces nouvelles. Argentine.

\section{Fleas from Neuquén Province (Argentina); description of 4 new taxa (Insecta, Sipho- naptera).}

SUMMARY. The collect of 973 Fleas on various small terrestrial mammals, from december 1987 till may 1988, in the Neuquén Province (Argentina), has given 20 taxa. Hectopsylla pascuali n. sp., Plocopsylla consobrina n. sp., Agastopsylla boxi gibbosa n. ssp., Chiliopsylla allophyla tonnii n. ssp. are described. Plocopsylla diana Beaucournu, Gallardo and Launay, 1986, Ctenoparia inopinata Rothschild, 1909, Neotyphloceras crassispina chilensis Jordan, 1936, Tetrapsyllus satyrus Beaucournu and Torrés-Mura, 1986, Ectinorus martini Lewis, 1976, are new from Argentina.

Key-words: Fleas. Siphonaptera. New species. Argentina.

La Province de Neuquén en Argentine n'avait encore pratiquement jamais été concernée par des études sur les Puces (Siphonaptera). Située entre 36 et 41 degrés de latitude Sud et centrée sur le $70^{\mathrm{e}}$ de longitude Ouest, elle est limitée

* Laboratoire de Parasitologie, Faculté de Médecine, Avenue du Pr Léon-Bernard, 35043 Rennes Cedex (France).

** Institut d'Estudis Avançats de les Illes Baleares (C. S. I. C.), 07071 Ciatat de Mallorca (Espagne).

*** Travail en partie réalisé grâce à une bourse du CONICET (Argentine).

Accepté le 5 juin 1989. 
à l'ouest par la frontière chilienne, au nord par la province de Mendoza, à l'est par celle de la Pampa et au sud-sud-est par celle du Rio Negro. Nous y avons prospecté une vingtaine de stations toutes localisées dans la partie sud, soit entre 39 et 41 degrés. Sans évoquer ici la faune des puces chiliennes, rappelons que les provinces de Mendoza et de Rio Negro ont fait l'objet de plusieurs et quelquefois importantes prospections (Jordan, 1942 ; Jordan, 1953 ; Smit, 1955 ; Smit, 1963 ; Gimenez, Ciccarelli et de la Barrera, 1964 ; Beaucournu et Gallardo, 1988).

Nous ne donnerons pas ici la description des biotopes mais une simple carte de situation de la zone prospectée (Carte 1). C’est une région montagneuse (nos

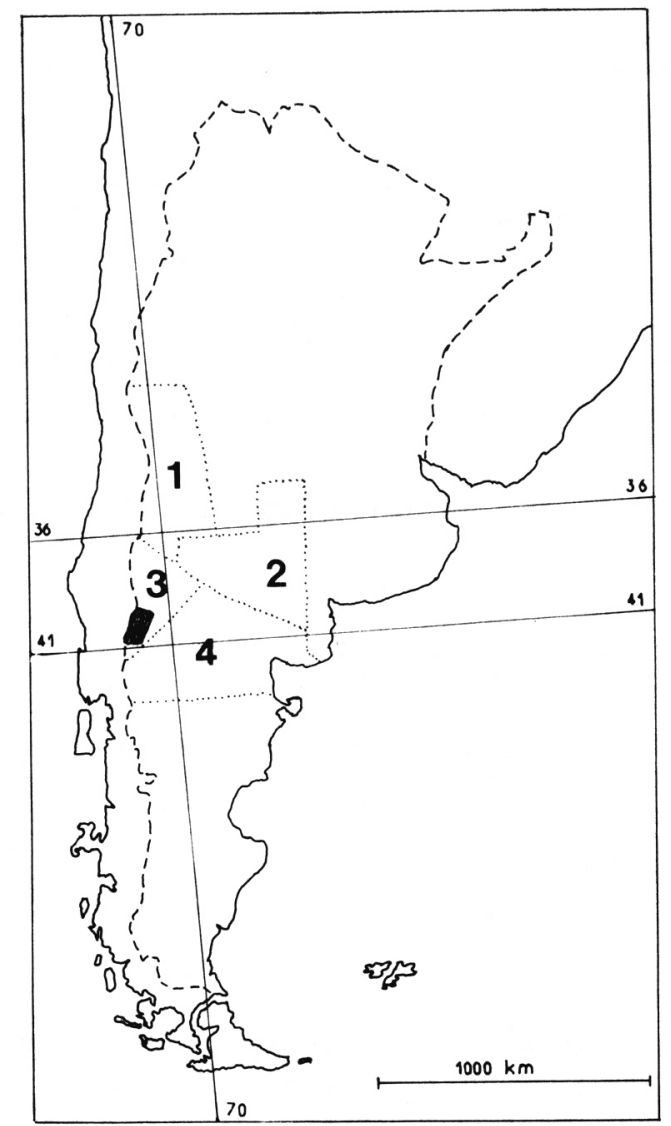

Carte 1 : Argentine et indication des 4 Provinces évoquées dans l'introduction.

1 : Mendoza ; 2 : La Pampa ; 3 : Neuquén (la zone de piégeage est en noir); 4 : Rio Negro.

captures s'étagent entre 700 et $1200 \mathrm{~m}$ d'altitude) mais de faciès variés : forêts andines (essentiellement à Nothophagus), maquis (matorral) à Embothrium, coteaux secs et prairies à plantes épineuses. 
Au total 973 siphonaptères ont été récoltés de décembre 1987 à avril 1988 provenant d'environ 325 petits mammifères : vingt taxa sont représentés, dont 2 espèces et 2 sous-espèces nouvelles pour la Science ; cinq autres sont nouveaux pour l'Argentine (tableau I).

Tableau I. - Puces récoltées dans le sud de la Province de Neuquén de décembre 1987 à avril 1988.

\begin{tabular}{|c|c|c|c|c|}
\hline & శే & q & Total & S. R. \\
\hline $\begin{array}{l}\text { Famille Pulicidae } \\
\text { 1. Hectopsylla pascuali n. sp. } \\
\text { Famille Stephanocircidae }\end{array}$ & 1 & 4 & 5 & 1 \\
\hline $\begin{array}{l}\text { 2. Sphinctopsylla ares } \\
\text { 3. Plocopsylla wolffsohni } \\
\text { Plocopsylla indet. } \\
\text { 4. Plocopsylla consobrina n. sp. } \\
\text { 5. Plocopsylla diana } \\
\text { 6. Barreropsylla excelsa }\end{array}$ & $\begin{array}{r}53 \\
32 \\
0 \\
3 \\
5 \\
6\end{array}$ & $\begin{array}{r}105 \\
41 \\
5 \\
1 \\
11 \\
14\end{array}$ & $\begin{array}{r}158 \\
73 \\
5 \\
4 \\
16 \\
20\end{array}$ & $\begin{array}{l}0,50 \\
0,78 \\
/ \\
1 \\
0,45 \\
0,43\end{array}$ \\
\hline $\begin{array}{l}\text { Famille Ceratophyllidae } \\
\text { 7. Nosopsyllus fasciatus } \\
\text { Famille Hystrichopsyllidae }\end{array}$ & 0 & 1 & 1 & 1 \\
\hline $\begin{array}{l}\text { 8. Ctenoparia inopinata } \\
\text { 9. Ctenoparia jordani } \\
\text { 10. Ctenoparia topali }\end{array}$ & $\begin{array}{r}49 \\
0 \\
31\end{array}$ & $\begin{array}{r}52 \\
5 \\
36\end{array}$ & $\begin{array}{r}101 \\
5 \\
67\end{array}$ & $\begin{array}{l}0,94 \\
/ \\
0,86\end{array}$ \\
\hline $\begin{array}{l}\text { Famille Ctenophthalmidae } \\
\text { 11. Agastopsylla boxi gibbosa n. ssp. } \\
\text { 12. Chiliopsylla allophyla tonnii } \mathrm{n} \text {. ssp. } \\
\text { 13. Neotyphloceras crassispina chilensis } \\
\text { 14. Neotyphloceras crassispina hemisus } \\
\text { Neotyphloceras crassispina ssp. } \\
\text { Famille Rhopalopsyllidae } \\
\text { 15. Tetrapsyllus tantillus } \\
\text { 16. Tetrapsyllus rhombus } \\
\text { 17. Tetrapsyllus satyrus } \\
\text { 18. Ectinorus martini } \\
\text { 19. Ectinorus onychius onychius } \\
\text { 20. Listronius fortis }\end{array}$ & $\begin{array}{r}30 \\
31 \\
7 \\
97 \\
3 \\
1\end{array}$ & $\begin{array}{r}38 \\
41 \\
6 \\
71 \\
3 \\
1\end{array}$ & $\begin{array}{r}68 \\
72 \\
13 \\
168 \\
6 \\
2\end{array}$ & $\begin{array}{l}0,8 \\
0,75 \\
1,16 \\
1,36 \\
/\end{array}$ \\
\hline
\end{tabular}

ÉTUde SystémAtiQue

Famille Pulicidae

1 - Hectopsylla pascuali n. sp.

Matériel de description : mâle holotype, femelle allotype, 3 femelles paratypes sur Chelemys macronyx (Rod., Cricet.), Arroyo Chapelco, province de Neuquén (Argentine), 9-XII-1987. 
Holotype, allotype et un paratype sont dans les collections du premier auteur (ultérieurement déposées au Laboratoire d'Entomologie du Muséum National d'Histoire Naturelle de Paris), 2 paratypes sont dans les collections d'entomologie de l'Institut d'Estudis Avançats de les Illes Baléares (C. S. I. C.), Ciutat de Mallorca (Espagne).

Nous sommes heureux de pouvoir dédier ce taxon au Dr Rosendo Pascual, Paléontologiste (Museo de la Plata), en respectueux hommage à ses travaux sur la faune des mammifères fossiles de l'Amérique du Sud.

\section{DESCRIPTION}

Espèce à tarse $\mathrm{V}$ pourvu de 4 soies latérales fortes : allex présent; index relativement épais (fig. \%, 8 et 13).

Mâle. Capsule céphalique à courbure presque régulière (fig. 1). Une forte soie pré-oculaire. Laciniae, un peu plus longue que le palpe maxillaire, atteignant les $4 / 5$ environ de la coxa I. Deux soies du segment II du tarse III dépassent l'apex du segment V. Sétation tergale (de I à VII) respectivement de 3, 3, 3, 2, 2,2 et 2 . Stigmates ronds. Sensilium : cf. fig. 3. Segment IX (fig. 3, 4) : Manubrium (M1) bien développé ; Basimère (P1), quadrangulaire ; Télomère (P3) bien développé, aussi long que la plus grande largeur de P1. Sternite IX bien caractérisé par son lobe ventral. Phallosome (fig. 5) caractéristique et bien séparable de celui de H. gracilis Mahnert, 1982 (fig. 6) qui en est proche par les autres caractères (en dehors des griffes tarsales (fig. 9, 10)).

Femelles. Capsule céphalique plus anguleuse que celle du mâle; sétation identique (fig. 2). Laciniae faisant près du double de la longueur du palpe maxillaire et dépassant la coxa I d'environ $1 / 3$. Tergite I avec un long processus distal. Métépimeron allongé, montrant un fin prolongement de l'angle postéro-dorsal comme chez $H$. gracilis (fig. 11 et 12) ; 4 soies latérales. Pattes comme chez le mâle. Sétation tergale réduite aux segments I et II (2 soies sur chaque) (fig. 11). Stigmates circulaires et relativement grands. Sensilium : cf. fig. 14. Sternite VII non distinctif comme il est de règle dans la Famille. Tergite VIII montrant 6 à 9 fortes soies insérées à sa face interne près de la marge postérieure (fig. 15). Spermathèque (fig. 16) apparemment sans caractère spécifique. Ducti non visibles sur nos exemplaires comme il est classique dans cette Famille.

Fig. 1-2. - Hectopsylla pascuali n. sp., capsule céphalique.

1 : mâle holotype ; 2 : femelle allotype.

FIg. 3-10. - Hectopsylla spp., mâles. 3:H. pascuali, holotype, sensilium et tergite IV; $4: d^{\text {o }}$, sternite IX ; $5: d^{0}$, apex du phallosome ; $6: H$. gracilis, paratype, apex du phallosome; 7 à 10 : griffes tarsales I et III de $H$. pascuali $(7,8)$ et de $H$. gracilis $(9,10)$.

Fig. 11-16. - Hectopsylla spp., femelles. 11: H. pascuali, allotype, tergite I et métépimeron ; $12: H$. gracilis, , paratype, $d^{0} ; 13: H$. pascuali, paratype, tarse III ; $14: d^{0}$, sensilium ; $15: d^{\circ}$, tergite VIII ; $16: d^{\circ}$, allotype, spermathèque. 

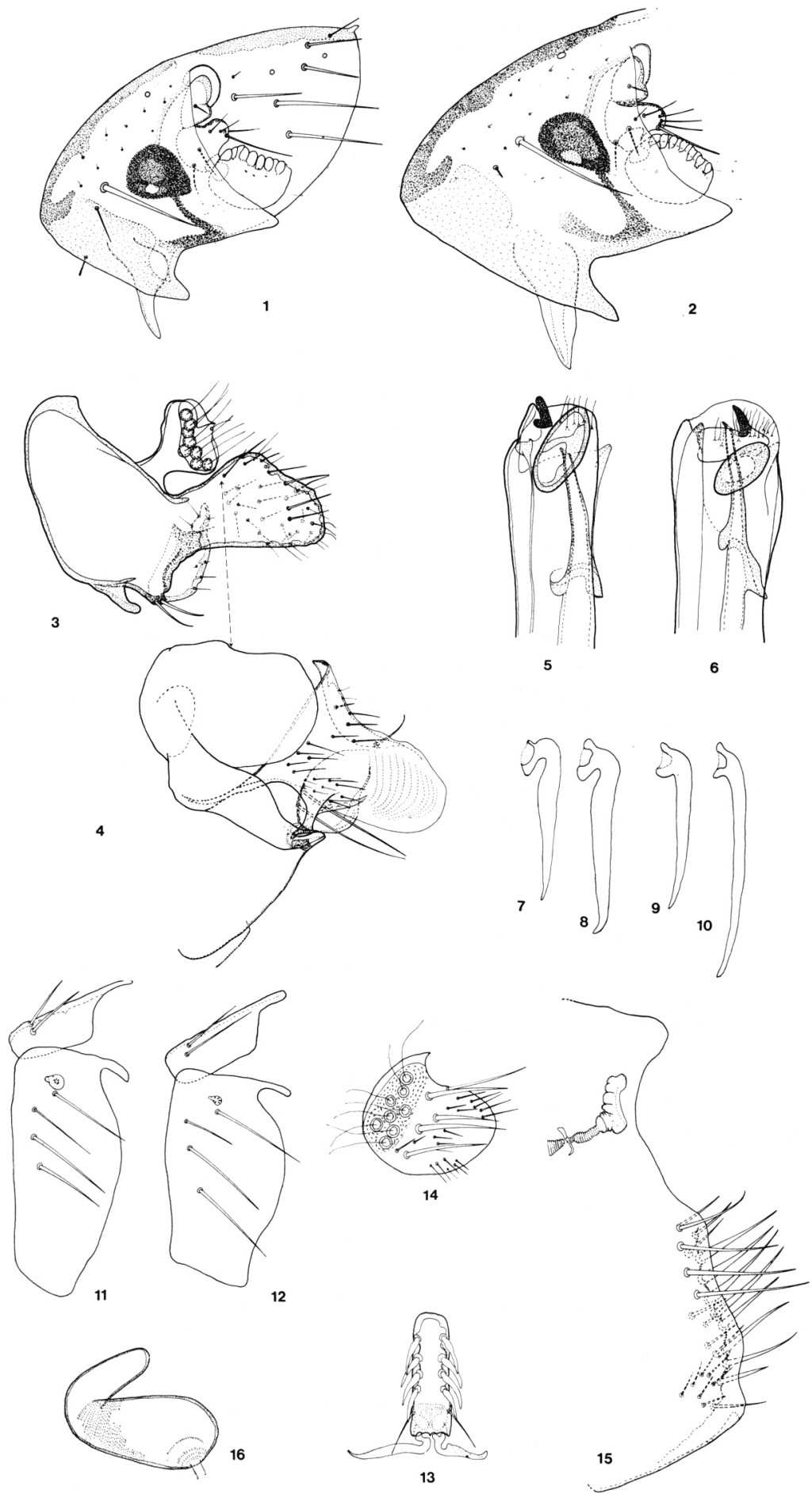
Dimensions (insectes montés) : mâle $1 \mathrm{~mm}$, femelle non gorgée 1,2 mm, femelle gorgée $2 \mathrm{~mm}$.

\section{Discussion}

Hectopsylla pascuali est très proche de gracilis, décrite de la province de Chubut sur la côte orientale de l'Argentine, mais également de suarezi Fox, 1929, eskeyi Jordan, 1933 et cypha Jordan, 1942.

$H$. pascuali n. sp. se sépare, dans les 2 sexes, des espèces précédentes par ses griffes tarsales. Chez le mâle, segment IX et phallosome, chez la femelle la conjonction du profil céphalique, de la chétotaxie et de la structure du métépimeron, permettent également l'identification.

Famille Stephanocircidae

2 - Sphinctopsylla ares (Rothschild, 1911)

Apparemment la plus répandue des Stephanocircidae dans la région étudiée : Lago Huechulafquen sur Rattus norvegicus, Akodon longipilis et Geoxus valdivianus; Cerro Chapelco sur Ak. longipilis; Laguna Verde sur le mème hôte; Lagos Curruhue sur $R$. norvegicus, A. olivaceus, Ak. longipilis, Oryzomys longicaudatus, Irenomys tarsalis; Lago Lolog sur Ak. longipilis; Quilanlahué sur le même hôte et Irenomys tarsalis ; Lago Norquinco sur O. longicaudatus et Ak. longipilis; Hua Hum sur Ak. longipilis; Ruca Malen sur ce même hôte, O. longicaudatus et Irenomys tarsalis; Volcan Huanqui Hue sur Ak. longipilis.

$S$. ares est connue du Chili comme de l'Argentine mais est limitée à la région chilio-andine semble-t-il.

Le sex-ratio est très déséquilibré ( 0,50 sur 158 exemplaires), mais ce phénomène semble toucher, à des degrés divers, la majorité des Craneopsyllinae.

\section{3 - Plocopsylla wolffsohni (Rothschild, 1909)}

Nombreuses récoltes de cette Plocopsylla. Notons toutefois que 2 prélèvements concernant des femelles seules, très proches de wolffsohni, n'y sont pas intégrées : des détails de structure et de pigmentation dans un cas, une spermathèque de type traubi Del Ponte (1968) dans l'autre, nous conduisent à ne pas leur attribuer de détermination spécifique.

Matériel examiné provenant de : Cerro Chapelco sur Ak. longipilis; Laguna Verde sur le même hôte ; Lagos Curruhue sur le même hôte, sur Ak. olivaceus et Irenomys tarsalis; Lago Norquinco sur Ak. longipilis; Ruca Malen sur le même hôte; Lago Quillen sur le même hôte.

$P$. wolffsohni a la mème répartition connue que les espèces précédentes, région du Rio Negro en Argentine et Chili central (terra typica: Valparaiso). Elle est nouvelle pour la région étudiée. 
4 - Plocopsylla consobrina n. sp.

Matériel de description : mâle holotype sur Akodon longipilis (Rod., Cricet.), Ruca Malen (Parc Naturel Nahuel Huapi), Province de Neuquén (Argentine), 16-IV-1988; femelle allotype, même hôte, Curruhue Grande, province de Neuquén, 21-IV-1988; 1 mâle paratype, même hôte et localité que l'holotype mais 17-IV-1988 ; 1 mâle paratype, même hôte, Pampa de Hui Hui, province de Neuquén, 28-IV-1988.

Holotype, Allotype et 1 paratype dans la collection du premier auteur ( $c$. supra), 1 paratype à Mallorca (cf. supra). Le nom montre la parenté étroite entre les divers membres de ce complexe.

\section{Description}

Capsule céphalique (fig. 17) : casque relativement étroit, sa plus grande largeur étant égale à la longueur de la dent dorsale de la cténidie correspondante. Cténidie frontale de 11 dents chez les mâles, 12 chez la femelle, la dent la plus longue étant la $5^{\mathrm{e}}$ (de haut en bas) chez les mâles, la $6^{\mathrm{e}}$ chez la femelle. Gena plus longue que large. Cténidie génale de 5 dents, la dent supérieure étant plus grêle et séparée de la suivante par un intervalle égal à la largeur de sa base. Soies du pédicelle antennaire courtes.

Prothorax (fig. 17) avec 2 rangées de soies. Cténidie prothoracique de 16 dents. 1 ou 2 soies du métathorax et des premiers tergites abdominaux semi-dressées. chez les mâles. 3 rangées de soies mésothoraciques et 2 pseudosetae, ces dernières bien sclérifiées; 2 rangées de soies métathoraciques. Griffes tarsales robustes. avec un sinus bien marqué entre index et allex.

Présence de spinules sur les tergites $\mathrm{I}$ à $\mathrm{V}$ : mâles respectivement 3 ou 4 , 2 à 4,1 à $3,2,1$; femelle, $4,4,3,2,1$ (aucune du côté droit sur ce tergite). Une seule soie antésensiliale, celle du mâle épaisse, relativement courte et flanquée dorsalement d'une soie vestigiale; la soie antésensiliale est, dans les deux sexes. sur un piédestal, celui du mâle étant particulièrement développé.

Segments modifiés mâles. Segment VIII (fig. 19) : tergite en oval allongé; sternite VIII à apex camus, bord ventral très échancré avec une zone échinulée. Segment IX (fig. 18) : tergite à bord dorsal doucement concave et plus court que le bord ventral distinctement incurvé. Processus interne (" digitiforme ") à base très large et à apex arrondi ne dépassant pas la marge dorsale du basimère. Processus fixe en angle obtus, l'apex distal élargi et strié. Télomère du groupe "wolffsohni-chiris », caractérisé par ses soies modifiées : l'externe bifide avec une partie médiane en bosse, striée, séparant ces deux pointes; l'apicale courte et peu sclérifiée avec seulement la pointe antérieure marquée et courbée. Sternite IX : bras distal inséré à distance de l'apex du bras proximal et nettement plus court que celui-ci. 2 soies médianes, très développées, en accent circonflexe; une soie apicale non modifiée mais assez longue; une soie subapicale aplatie et coudée, non sclérifiée. Apex du tubus interior coudé. 

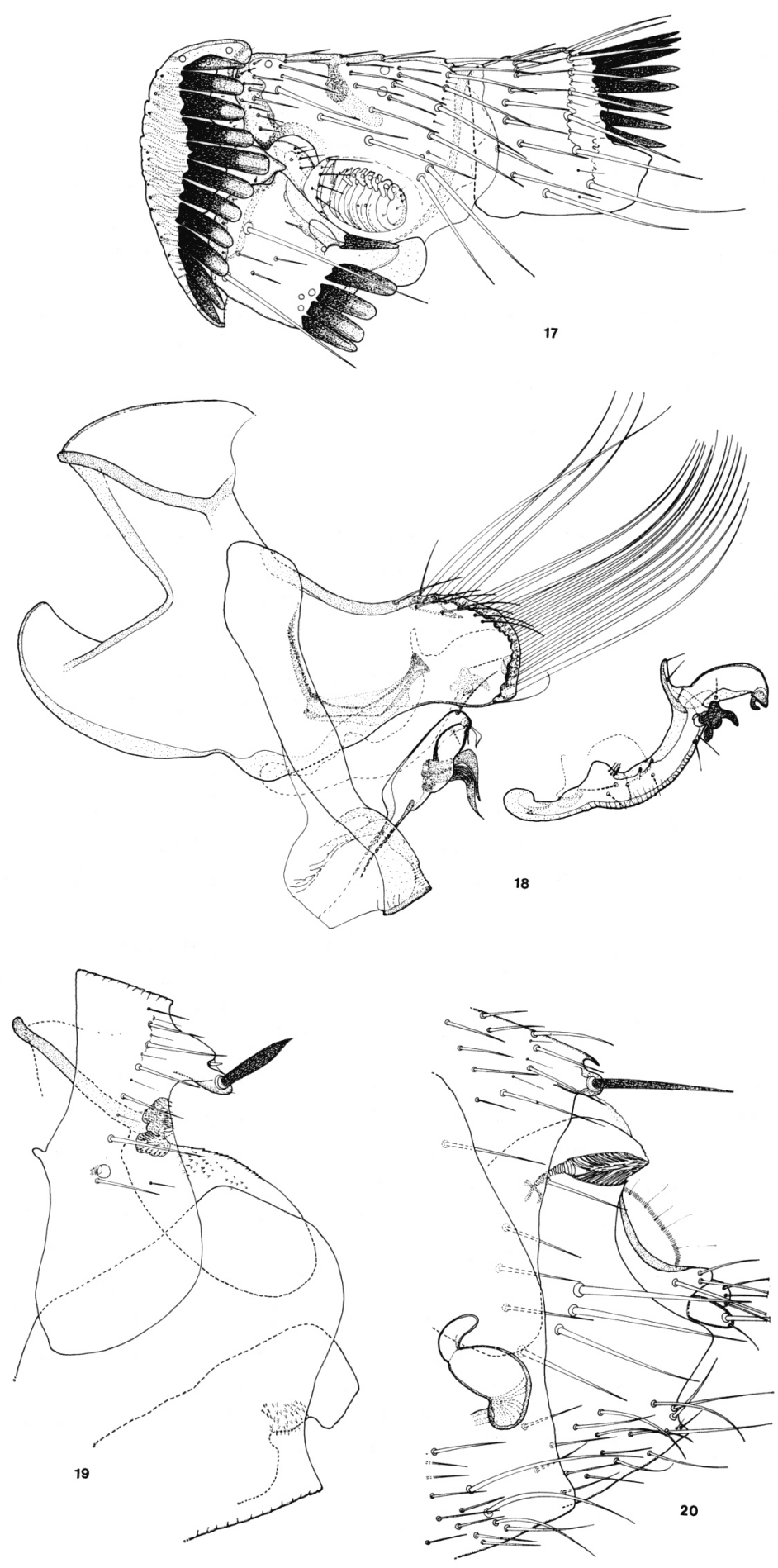
Segments modifiés femelles et spermathèque (fig. 20). Sternite VII sans caractères particuliers, portant comme le tergite VIII, quelques soies courbées, dans leur partie ventrale. Stigmate du t. VIII, allongé. Stylet anal environ deux fois plus long que large. Spermathèque : bulga arquée ventralement dans son tiers distal; hilla classique sans prolongement à l'intérieur de la bulga.

Dimensions (insectes montés) ; mâles : 2,1 mm (sans les soies distales du basimère), femelle : 2,5 .

\section{Discussion}

Flocopsylla consobrina appartient au complexe " wolffsohni-chiris » (cf. Beaucournu et Gallardo, 1977) qui regroupe actuellement, outre ces trois espèces, fuegina Beaucournu et Gallardo, 1977, reigi B. et G., 1977, diana Beaucournu, Gallardo et Launay, 1986, athena Schramm et Lewis, 1987, sp. nova Beaucournu et Gallardo, sous presse. $P$. diana est tout à fait à part, grâce à sa cténidie frontale très développée. $P$. chiris est écartée par de nombreux détails des genitalia. Toutes les autres espèces présentent (chiris également d'ailleurs) un processus fixe non élargi; la plus proche serait, hormis ce caractère, $P$. fuegina, qui se distingue, entre autres, par l'implantation du processus interne, la forme des soies modifiées du télomère et la gracilité relative de la soie antesensiliale.

5 - Plocopsylla diana Beaucournu, Gallardo et Launay, 1986

$P$. diana est, dans l'état actuel des espèces connues, imméđiatement caractérisée dans les deux sexes par le grand développement de sa cténidie frontale. Elle fut décrite du Chili (mâle holotype sur Rhyncholestes raphanurus (Marsupiala) à Palomar, Chiloë ; femelle allotype sur Akodon longipilis (Rod.), Parque. Nahuelbuta, Malleco, ces deux stations étant distantes de quelques $500 \mathrm{~km}$ ).

Nous en avons retrouvé 16 exemplaires dans 7 stations : Lago Huechulafquen sur $A k$. longipilis; Cerro Chapelco sur le même hôte; Laguna Verde sur le même hôte; Lagos Curruhue sur Dromiciops australis (Marsup.); Lago Lolog sur $A k$. longipilis; Hua Hum sur le même hôte ; Ruca Malen sur le même hôte.

Sans vouloir préjuger de sa spécificité notons que le seul Dromiciops parasité hébergeait la moitié de nos exemplaires.

$P$. diana est nouvelle pour l'Argentine.

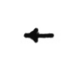

FIG. 17. - Plocopsylla consobrina n. sp., mâle holotype, capsule céphalique et prothorax.

FIG. 18. - Plocopsylla consobrina, mâle holotype, segment IX (et apophyse du tergite VIII).

FIG. 19-20. - Plocopsylla consobrina. 19 : segments VII et VIII d'un mâle paratype; 20 : segments terminaux et spermathèque de la femelle allotype. 
6 - Barreropsylla excelsa Jordan, 1953

Cette originale espèce fut décrite de la province du Rio Negro où elle fut successivement retrouvée par Smit (1955), Smit (1963), Beaucournu et Gallardo (1988) : c'est la seule zone de répartition publiée actuellement.

Nous la citons des stations suivantes : Lagos Curruhue sur Akodon longipilis, Oryzomys longicaudatus; Lago Norquinco sur O. longicaudatus; Ruca Malen sur Ak. longipilis; Lago Quillen sur Akodon sp.

Famille Ceratophyllidae

7 - Nosopsyllus fasciatus (Bosc d'Antic, 1800)

Puce cosmopolite avec le rat Rattus norvegicus, sporadique dans la région qui nous occupe. Elle est déjà, en particulier, signalée de la province du Rio Negro. Une seule capture dans nos relevés : San Martin de los Andes sur Akodon.

Famille Hystrichopsyllidae

8 - Ctenoparia inopinata Rothschild, 1909

Décrite sur une femelle de Valparaiso, ce sexe a été retrouvé depuis à plusieurs reprises ; par contre le mâle de cette espèce n'a été récolté et figuré que tout récemment (Beaucournu et al., 1986). Toutes ces stations concernaient le Chili.

Nous avons trouvé $C$. inopinata en abondance dans la Province de Neuquén : Lago Huechulafquen sur Akodon longipilis et Geoxus valdivianus; Cerro Chapelco sur Ak. longipilis; Laguna Verde sur le même hôte; Termes de Lauhenco sur le même hôte; Lagos Curruhue sur le même hôte et sur O. longicaudatus; Lago Lolog sur Ak. longipilis; Quilanlahué sur le même hôte; Lago Norquinco sur le même hôte; Hua Hum sur le même hôte ; Ruca Malen sur le même hôte.

A noter que 99 exemplaires sur 101 proviennent d'Akodon longipilis.

C. inopinata est nouveau pour l'Argentine.

9 - Ctenoparia jordani Smit, 1955

Espèce mal connue, décrite d'Argentine (Rio Negro), récemment retrouvée au Chili : Valdivia (Beaucournu et al., 1986). Elle est peu abondante dans nos prélèvements : Lagos Curruhue sur Akodon longipilis; Ruca Malen sur le même hôte.

Elle est nouvelle pour la province de Neuquén.

10 - Ctenoparia topali Smit, 1963

Ce taxon a été décrit du Rio Negro sur un mâle, puis signalé du Chili (Beaucournu et al., 1986); la femelle vient d'être décrite, de ce dernier pays également (Beaucournu et al., 1988). 
Nous la signalons des stations suivantes : Laguna Verde sur Ak. longipilis; Lagos Curruhue sur ce même hôte, Ak. olivaceus, O. longicaudatus, Irenomys tarsalis; Lago Norquinco sur Auliscomys micropus et O. longicaudatus; Ruca Malen sur Ak. longipilis, O. longicaudatus et Irenomys tarsalis; Lago Quillen sur Akodon sp.

Cette espèce vient en deuxième position par sa fréquence, pour le genre Ctenoparia. Notons que deux stations ont livré les 3 Ctenoparia citées et qu'un lot de 8 Ak. longipilis du même gîte (mais dont les parasites furent mêlés) a donné ces trois espèces. Leur sympatrie est donc bien démontrée (Beaucournu et al., 1986 ; Beaucournu et Gallardo, 1988).

C. topali est nouvelle pour la province de Neuquén ce qui élargi sa dispersion connue en Argentine puisque le seul exemplaire jusqu'à ce jour signalé de ce pays était l'holotype d'El Bolson, dans l'extrême sud-ouest du Rio Negro.

Famille Ctenophthalmidae

11 - Agastopsylla boxi gibbosa n. ssp.

\section{Matériel De Description}

Mâle holotype, femelle allotype, 1 mâle paratype sur Akodon longipilis (Rod., Cricet.), Lago Huechulafquen, province de Neuquén (Argentine), 12 et 13-XII-1987; 1 mâle paratype sur Ak. longipilis, Rio Curruhue, $6 \mathrm{~km}$ de Junin de los Andes, Neuquén, 8-XII-1987 ; 1 mâle paratype sur Ak. xanthorhinus, même endroit, même date; 1 femelle paratype sur Oryzomys longicaudatus, Rio Quilquihue, Neuquén, 9-XII-1987; 1 femelle paratype sur Auliscomys micropus, Cerro Chapelco, Neuquén, 17-XII-1987; 2 mâles paratypes sur Ak. longipilis, Lago Lolog, Neuquén, 21 et 22-XII-1987; 5 mâles et 5 femelles paratypes sur Ak. longipilis, Lagos Curruhue, Neuquén, 21 au 24-IV-1988; 1 femelle paratype sur Ak. olivaceus, même endroit, 22-IV-1988; 2 femelles paratypes sur Ak. longipilis, Lago Norquinco, Neuquén, I-V-1988; 1 mâle paratype sur Ak. olivaceus, même endroit, même date; 1 mâle paratype sur Auliscomys micropus, même endroit, 3-V-1988 ; 1 mâle, 3 femelles paratypes sur Oryzomys longicaudatus, même endroit, 1-V-1988 et 3-V-1988; 5 mâles, 3 femelles paratypes sur Ak. longipilis, Pampa Neuquén, 26-28-IV-1988 ; 1 mâle, 3 femelles paratypes sur hôte indéterminé, Rio Alumine, de Hui Hui, Neuquén, 4-V-1988.

Dépôt de l'holotype et de l'allotype : cf. supra; les paratypes sont répartis entre les deux Instituts précédemment cités, à l'exclusion de 3 couples respectivement déposés au British Museum, au Muséum d'Histoire Naturelle de Genève et dans la collection R. E. Lewis (Ames, Iowa).

Le nom (de gibbosus : bossu) évoque la variation particulière du sternite IX du mâle de ce taxon. 


\section{Description}

Immédiatement rattaché à la forme nominative par la plage de soies du tergite VII chez le mâle et par la structure des ducti chez la femelle.

Uniquement séparable par la conformation du sternite IX du mâle (fig. 21) dont le bord antérieur du bras distal montre une nette convexité associée à un apex plus étroit. Chez A. boxi boxi Jordan et Rothschild, 1923, les 2 bords du bras distal sont doucement courbés et parallèles, l'apex étant, pratiquement, de même largeur que ce bras (fig. 22). Par ailleurs, les soies apicales de ce sternite sont plus larges que les sous-jacentes chez $A$. b. gibbosa, ce qui n'est pas le cas chez $A$. b. boxi.

Les femelles des deux sous-espèces ne semblent pas différenciables.

Actuellement $A$. boxi boxi est connue d'Argentine : provinces de Chubut (terra typica) et du Rio Negro (Smit, 1955 ; Smit, 1963) où nous l'avons retrouvé (Beaucournu et Gallardo, 1988) et du sud du Chili (Smit et Rosicky, 1972).

12 - Chiliopsylla allophyla tonnii n. ssp.

\section{MATÉRIEL DE DESCRIPTION}

Mâle holotype, femelle allotype et 1 femelle paratype sur Akodon longipilis (Rod., Cricet.), Lago Huechulafquen, province de Neuquén (Argentine), 10-XII-1987; 1 mâle, 3 femelles paratypes sur Ak. longipilis, Cerro Chapelco, Neuquén, 16-17-XII-1987; 2 femelles paratypes sur Ak. longipilis, Termes de Lauhenco, Neuquén, 19-XII-1987 ; 1 femelle paratype sur Oryzomys longicaudatus, Lagos Curruhue, Neuquén, 13-II-1988; 1 mâle, 14 femelles paratypes sur Dromiciops australis, même endroit, 14-II-1988; 2 mâles, 14 femelles paratypes sur Ak. longipilis, même endroit, 20-24-IV-1988; 1 femelle sur Ak. longipilis, Ruca Malen, Neuquén, 17-IV-1988.

Holotype, allotype, cf. supra ; paratypes, dépôts dans les collections dépendantes des deux auteurs.

Ce nouveau taxon est dédié à notre collègue paléontologiste le Dr Eduardo Tonni (Museo de la Plata) en hommage et en remerciement.

\section{Description}

Pour ce taxon, de nouveau, seules les genitalia mâles sont caractéristiques. La partie basale du télomère apparaît moins élancée, ceci étant lié au développement et à l'allongement de l'apex de cette pièce chez Ch. a. tonnii (fig. 23, 24).

FIG. 21-22. - Agastopsylla boxi ssp., sternite IX du mâle. 21 : Agastopsylla boxi gibbosa n. ssp., holotype ; 22 : A. boxi boxi, Cerro Microondas (Rio Negro), Argentine (M. H. Gallardo rec.). FIG. 23. - Chiliopsylla allophyla tonnii n. ssp.,
mâle holotype, segment IX.

FIg. 24-25. - Chiliopsylla allophyla ssp., télomère (contour et insertion des soies internes apicales) 24 : Ch. allophyla tonnii, paratype, Lagos Curruhue (Neuquén), Argentine; $25:$ Ch. allophyla allophyla, Fundo la Dehesa (Valdivia), Chili (M. H. Gallardo rec.). 
PUCES D'ARGENTINE

501
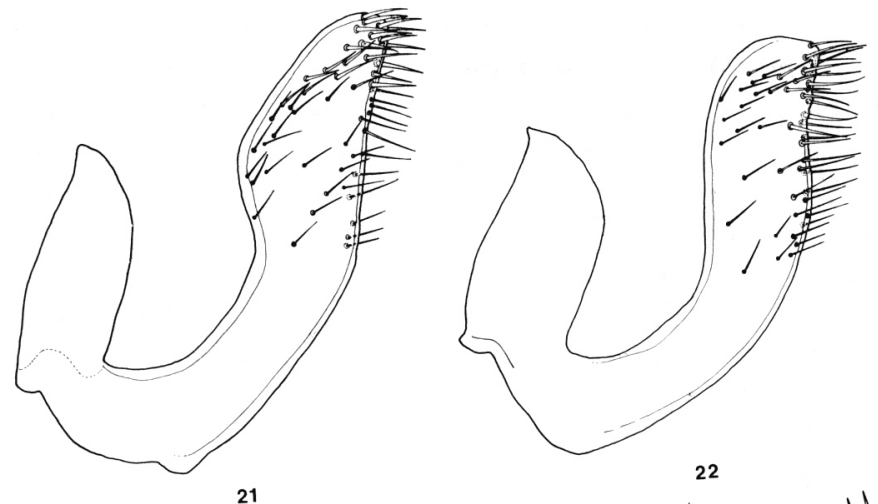

21

22
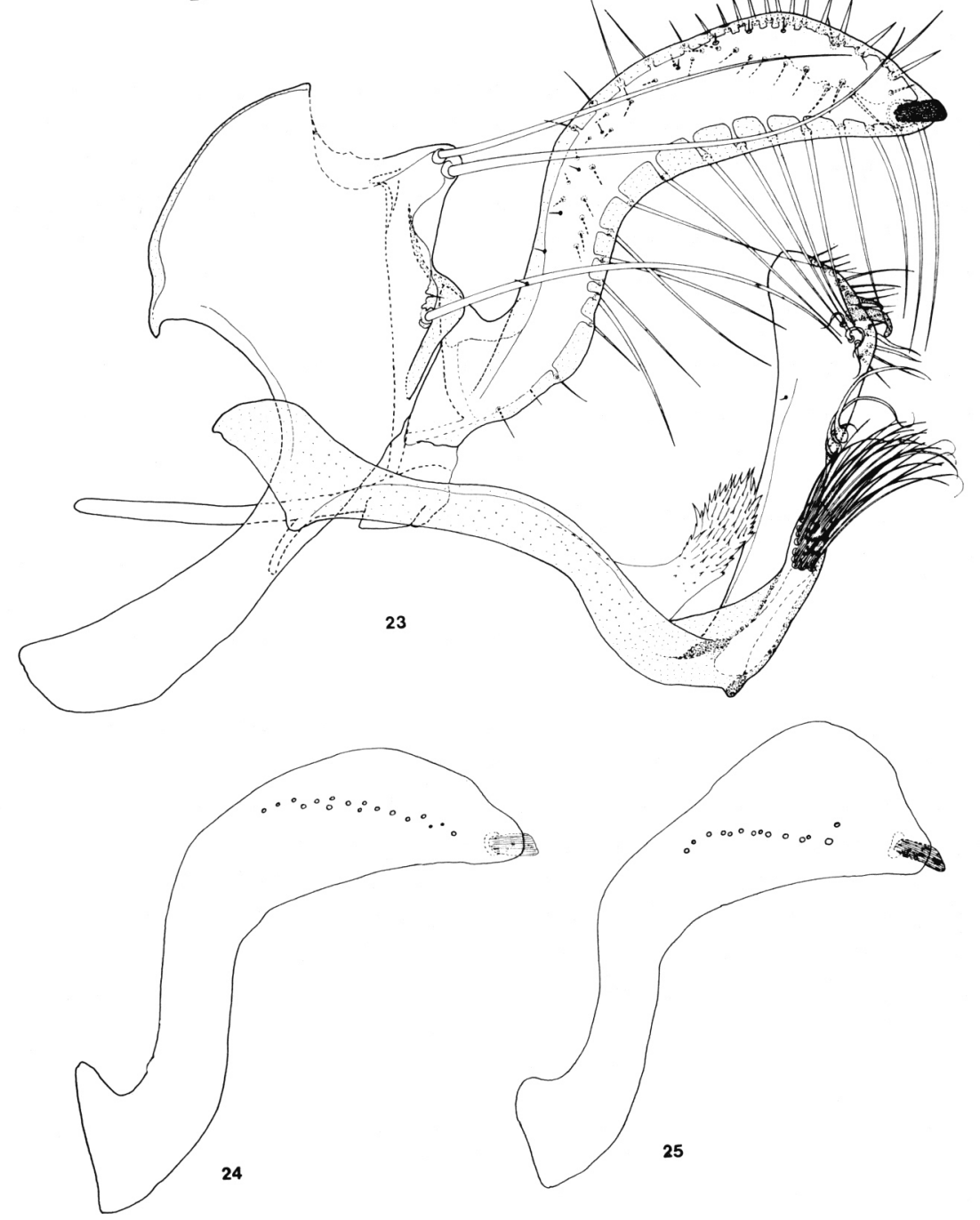
La comparaison avec un mâle chilien de la province de Valdivia appartenant à la forme nominative (fig. 25) ne permet de déceler aucune autre différence, taxonomiquement valable, ni dans la chétotaxie, ni dans les structures pourtant complexes du sternite IX et du phallosome. Les femelles sont indifférenciables.

La répartition de Chiliopsylla, genre monospécifique, est cantonnée au Chili et à l'ouest de l'Argentine. Ch. a. allophyla (Rothschild, 1908) est connue du Chili, qui est terra typica, et d'Argentine (Smit, 1955).

Hopkins et Rothschild (1966) suggéraient la possibilité d'une inféodation de cette puce à Dromiciops (Marsupiala). Cette spécificité, si elle existe, est manifestement peu stricte.

Notons enfin le très fort déséquilibre du sex-ratio dans notre matériel, 5 mâles seulement sur 45 exemplaires (s. r. $=0,12$ !).

- Neotyphloceras crassispina ssp.

Excepté trois exemplaires (mâles) de cette espèce largement répandue, nous considérons les populations de Neuquén comme essentiellement hybrides entre N. c. crassispina Rothschild, 1914 et N. c. hemisus Jordan, 1936. Nous avons toutefois eu la surprise dans l'un des gîtes (celui des Lagos Curruhue) d'obtenir, en dehors des exemplaires intermédiaires, 1 mâle typique de $N$. c. chilensis Jordan, $1936^{1}$ et un autre d'hemisus. L'autonomie de ce dernier taxon ne nous semble pas, d'ailleurs, évidente.

13 - Neotyphloceras crassispina chilensis Jordan, 1936

Lagos Curruhue (Lago Curruhue Chico) sur Octodon bridgesi.

Ce taxon est nouveau pour l'Argentine.

14 - Neotyphloceras crassispina hemisus Jordan, 1936

Lagos Curruhue (Lago Curruhue Grande) sur Akodon longipilis ; Pampa de Hui Hui sur le même hôte.

Ce taxon était déjà signalé de la province de Neuquén (Hopkins et Rothschild, 1966).

- Neotyphloceras cr. crassispina $\times$ cr. hemisus (ou N. crassispina ssp. femelles seulement, stations indiquées par un astérisque).

Rio Curruhue* sur Akodon longipilis et $A k$. xanthorhinus ; Rio Quilquihué sur O. longicaudatus ; Arroyo Chapelco sur Chelemys macronyx et Ak. longipilis ; Lago Huechulafquen sur Ak. longipilis ; San Martin de los Andes* sur Akodon sp. ; Cerro Chapelco* sur Ak. longipilis ; Laguna Verde* sur Rattus norvegicus ; Lagos Curruhue sur Ak. longipilis, Akodon sp., Octodon bridgesi ; Quilanlahué* sur Ak. longipilis ; Lago Norquinco sur Ak. longipilis, Ak. olivaceus ; Ruca Malen* sur Ak. longipilis.

1. Nous ne tentons pas sur ces populations "frontières "l'identification subspécifique des femelles (cf. Smit, 1968), les critères étant assez discrets et variables. 
FAMille Rhopalopsyllidae

15 - Tetrapsyllus (Tetrapsyllus) tantillus (Jordan et Rothschild, 1923)

Nombreux exemplaires provenant de : Rio Quilquihué sur Oryzomys longicaudatus ; Arroyo Chapelco sur Akodon longipilis et Auliscomys micropus ; Lago Huechulafquen sur Akodon longipilis; San Martin de los Andes sur le même hôte ; Cerro Chapelco sur le même hôte; Laguna Verde sur le même hôte; Lagos Curruhue sur le même hôte, sur Akodon olivaceus, O. longicaudatus et Auliscomys micropus ; Lago Norquinco sur Akodon longipilis, O. longicaudatus et A. micropus.

Cette puce est nouvelle pour la province mais est connu de divers points voisins en Argentine (Rio Negro) et du centre du Chili (région de Valparaiso en particulier).

16 - Tetrapsyllus (T.) rhombus Smit, 1955

Nombreux exemplaires provenant de : Lago Huechulafquen sur Rattus norvegicus et Akodon longipilis ; Laguna Verde sur Akodon longipilis ; Lagos Curruhue sur Octodon bridgesi, Ak. longipilis, Ak. olivaceus, Aconaemys sp.; Hua Hum sur Ak. longipilis ; Ruca Malen sur ce même hôte ; Pampa de Hui Hui sur ce même hôte et Oryzomys longicaudatus.

La répartition connue est la même pour l'Argentine que l'espèce précédente et elle est donc nouvelle pour la province; elle est également signalée du Chili (Smit, 1987).

\section{7 - Tetrapsyllus (Phylliver ?) satyrus Beaucournu et Torrés-Mura, 1986}

Une petite série de 13 exemplaires ( 7 mâles, 6 femelles) récoltée aux Lagos Curruhue sur Akodon longipilis, Octodon bridgesi et Aconaemys sp. : ce dernier hôte a livré 5 de ces puces (et également le mâle et la femelle types).

Cette espèce est nouvelle pour l'Argentine. Elle est connue du Chili dans les provinces voisines de Cautin (terra typica) et Malleco. Son occurrence était d'autant plus vraisemblable en territoire argentin que le matériel-type provient du Paso Reigolil à la frontière même entre ces 2 pays, à $50 \mathrm{~km}$ environ (à vol d'oiseau !) au nord de nos gîtes.

T. salyrus est immédiatement caractérisé dans le genre Tetrapsyllus par divers caractères de chétotaxie, la structure du phallosome, la conformation respectives des sternites VI et VII de la femelle, la structure des ducti. Des petites différences sont notables entre les exemplaires de Cautin ( $c$. Beaucournu et TorrésMura, 1986) et ceux de Neuquén : taille du sinus du sternite VII (fig. 26), forme de la spermathèque ${ }^{2}$ (fig. 27 ), forme du lobe latéral du phallosome (fig. 28 à 31). Il nous semble prématuré d'y voir une sous-espèce autonome. De même son rattachement au sous-genre Phylliver Smit, 1987 est tout à fait hypothétique.

2. Le ductus spermathecae orné de spicules semble un caractère original pour le genre Tetrapsyllus. 


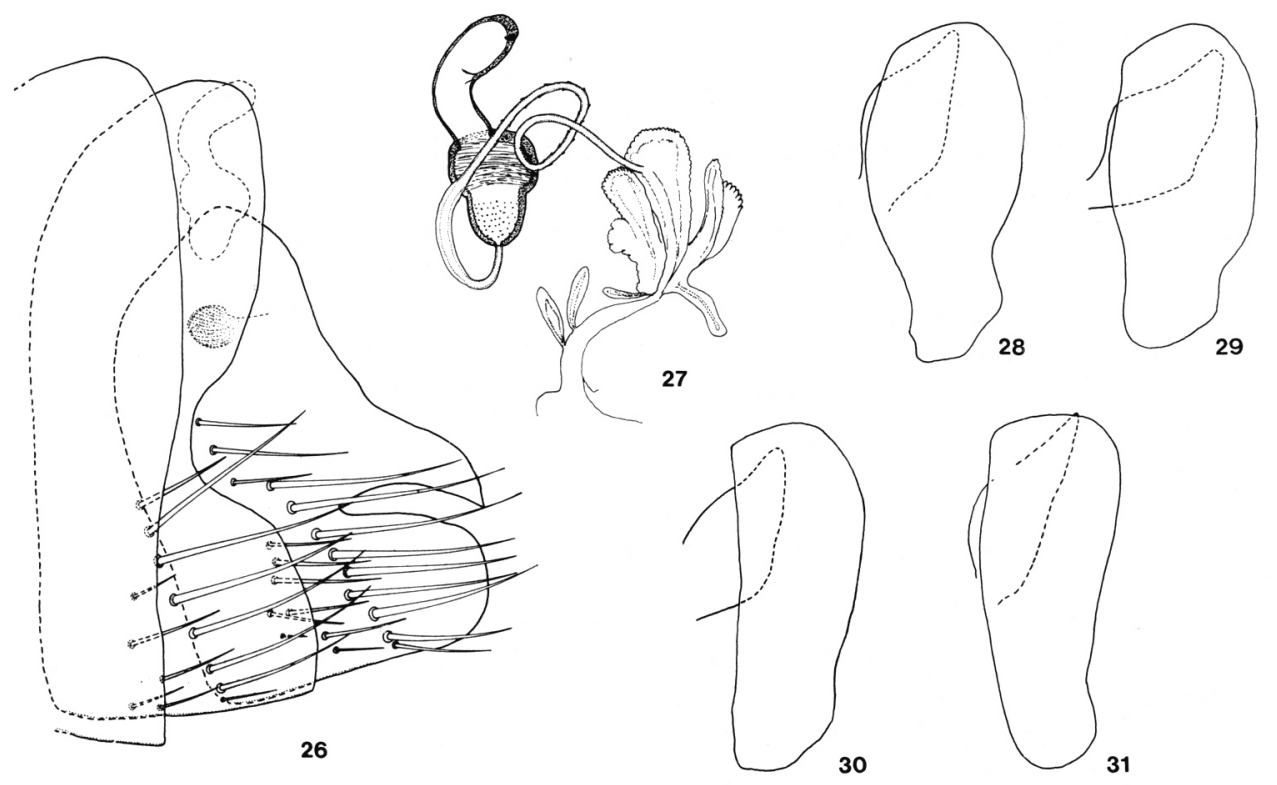

Fra. 26-31. - Tetrapsyllus satyrus. 26 : sternites V à VII, femelle, Lagos Curruhue (Neuquén) Argentine; 27 : spermathèque et ducti, $d^{\mathbf{0}} ; 28$ à 31 : lobe latéral du phallosome ; 28 : Lagos Curruhue (Neuquén); $29: d^{0} ; 30$ : Victoria (Cautin), Chili ; 31 : Parco National Tolhueco (Malleco), Chili (M. H. Gallardo rec.).

\section{8 - Ectinorus (Ectinorus) martini Lewis, 1976}

C'est une puce abondante dans nos récoltes : Arroyo Chapelco sur Chelemys macronyx ; Lago Huechulafquen sur Ak. longipilis et Geoxus valdivianus ; Lagos Curruhue sur Ak. longipilis, Oryzomys longicaudatus, Octodon bridgesi et Aconaemys sp.; Quilanlahué sur Irenomys tarsalis ; Lago Norquinco sur Aconaemys sp.; Pampa de Hui Hui sur Ak. longipilis et O. longicaudatus ; Lago Quillen sur Akodon sp.

Nos mâles, tant chiliens qu'argentins montrent de légères différences avec l'holotype. En particulier, l'apex du sternite IX, sans doute pour un problème d'orientation, ne paraît que rarement bifide.

Les 168 exemplaires récoltés montrent un sex-ratio de 1,36 ce qui est assez rarement noté chez les puces, où en général, les femelles sont légèrement en excès. Une exception du même ordre se rencontre, par exemple, chez Doratopsylla dasycnema et il semble bien qu'il s'agisse en fait d'une différence liée au comportement de l'imago (Beaucournu, 1977).

E. martini est nouveau pour l'Argentine.

19 - Ectinorus (Ichyonus) onychius onychius (Jordan et Rothschild, 1923)

Espèce rare dans nos récoltes, présente seulement dans 2 stations où elle 
n'est représentée que par 6 exemplaires : Lagos Curruhue sur Ak. longipilis et $A k$. olivaceus ; Lago Norquinco sur Ak. longipilis.

Nos stations remontent quelque peu vers le Nord la répartition connue de cette sous-espèce limitée, pour le moment, à l'Argentine.

20 - Listronius fortis (Jordan et Rothschild, 1923)

Cette rare espèce ne figure dans nos récoltes que par un couple : Cerro Chapelco sur Ak. longipilis ; Lago Norquinco sur le même hôte.

L. fortis est, actuellement, seulement connu d'Argentine. Nos captures sont nouvelles pour la province de Neuquén.

\section{RÉFÉRENCES}

Beaucournu J. C. : Notes sur les Hystrichopsyllidae (Siphonaptera) de la Faune française. (Première partie : Répartition, Biologie) (suite). Ann. Soc. Entomol. Fr. (N. S.), 1977, 13, 21-52.

Beaucournu J. C., Gallardo M. H. : Quelques nouvelles puces du Chili (Siphonaptera), parasites de Ctenomys (Rod., Octodontidae). Bull. Soc. Path. Exot., 1977, 70, 438-450.

Beaucournu J. C., Gallardo M. H. : Puces nouvelles d'Argentine (Insecta, Siphonaptera). Rev. Suisse Zool., 1988, \$5, 99-112.

Benucournu J. C., Gallardo M. H. : Contribution à la Faune du Chili ; Puces nouvelles de la moitié Nord (Insecta, Siphonaptera). Bull. Soc. Entomol. Fr., 1990, sous presse.

Beaucournu J. C., Gallardo M. H., Launay H. : Puces (Siphonaptera) nouvelles ou peu connues du Chili : description de Plocopsylla diana n. sp. (Stephanocircidae). Ann. Parasitol. Hum. Comp., 1986, 61, 359-366.

Beaucournu J. C., Torres-Mura J. C. : Un Tetrapsyllus nouveau du Chili (Siphonaptera, Rhopalopsyllidae). Rev. Fr. Entomol. (N. S.), 1986, 8, 9-12.

Beaucournu J. C., Torres-Mura J. C., Gallardo M. H. : Description de la femelle de Ctenoparia topali Smit, 1963 et clef dichotomique du genre Ctenoparia Rothschild, 1909 (Siphonaptera, Hystrichopsyllidae). Ann. Parasitol. Hum. Comp., 1988, 63, 380-383.

Gimenez D. F., Ciccarelli C. A., Barrera J. M. De la : Siphonaptera de Mendoza. Ann. Soc. Cient. Argent., 1964, 178, 125-139.

Hopkins G. H. E., Rothschild M. : An illustrated catalogue of the Rothschild collection of Fleas (Siphonaptera) in the British Museum (Natural History). Vol. IV : Hystrichopsyllidae. British Museum (Nat. Hist.), 1966, viI + 549 p.

Jordan K. : On the Siphonaptera collected by Dr J. M. de la Barrera in the province of Mendoza during 1939. Rev. Inst. Bact. "Dr Carlos G. Malbran ", 1942, 10, 401-460.

Jordan K. : On a very remarkable flea from Argentina. Bull. Br. Mus. (Nat. Hist.), Entomol., $1953,3,179-185$.

Sмiт F. G. A. M. : Siphonaptera from Bariloche, Argentina, collected by Dr J. M. de la Barrera in 1952-1954. Trans. R. Entomol. Soc. London, 1955, 10, 319-340.

Smit F. G. A. M. : The Zoological Results of Gy. Topal's collectings in South Argentina. 4. Siphonaptera. Ann. Hist.-Nat. Mus. Nation. Hungar., Zool., 1963, 55, 421-433.

Sмгт F. G. A. M. : Siphonaptera taken from formalin-traps in Chile. Zool. Anzeig., 1968, 180, $220-228$.

Sмiт F. G. A. M. : An illustrated catalogue of the Rothschild collection of Fleas (Siphonaptera) in the British Museum (Natural History). Vol. VII : Malacopsylloidea (Malacopsyllidae and Rhopalopsyllidae). British Museum (Nat. Hist.), 1987, 380 p.

Smit F. G. A. M., Rosicky B. : Some Siphonaptera from Chile. Folia Parasitol. (Praha), 1972, $19,365-368$. 